\title{
Pharmacological Research of Schisandra Chinensis(Turcz.) Baill.
}

\author{
Zhidong Hou, Guangyu Xu, Xiao Han, Guangxin Yuan, Yu Sheng, Hongyu Li, \\ Jingbo Sun, Manli Wang, Nanxi Zhao, Peige Du *, Liping An * \\ College of Pharmacy, Beihua University; Jilin, Jilin 132013, China
}

alp960@126.com

\begin{abstract}
Keywords: Schisanda Chinensis Baill, pharmacological activity, chemical composition.
Abstract. Schisanda Chinensis Baill is one of the most commonly used in Chinese folk medicine, it belongs to the magnoliaceae plants, named Chinese prickly ash, its medicinal part is fruit and traditional Chinese medical theory have already implicated that its acid, sweet, warm, lungs, heart, kidney, with fluid convergence lung, kidney nourishing the heart, the effect of convergence Guse. The author mainly studied chemical constituents of Chinese magnoliavine and its pharmacological activities in recent years, his research has carried on the summary and analysis for the future, the study of Schisanda Chinensis Baill provides some references for the development and utilization.
\end{abstract}

\section{Introduction}

There are the other names of Schisandra chinensis(Turcz.) Baill such as Xuan, Five plum blossom and Mountain pepper. Schisandra chinensis(Turcz.) Baill belongs to the magnoliaceae plants which is the dried fruit. The former is commonly known as the North Schisandra chinensis, and the latter is considered as the South Schisandra. Schisandra chinensis is a kind of traditional Chinese medicine, its acid, sweet, warm, lungs, heart, kidney, with fluid convergence lung, kidney nourishing the heart, the effect of convergence Guse such as [1]. Chinese Schisandra are more than 20 species, of which there are 10 species of Kadsura. Now, the most commonly used is the Schisandra chinensis, followed by the Schisandra sphenanthera. And the composition of Fructus Schisandra chinensis mainly contains lignans, polysaccharides, essential oil, fatty acid, vitamin and amino acid. [2]. Modern medical research shows that Schisandra has the effect of protecting liver, lowering blood glucose, anti-tumor, antioxidant and protecting the central nervous system.

\section{Pharmacological Research of Schisandra Chinensis(Turcz.) Baill..}

Liver protective effect. Schisandra chinensis(Turcz.) Baill can reduce the level of the liver transaminase and increase the P-450 content of liver cells, it also can improve liver protein synthesis, increase serum albumin index, as well as promote gluconeogenesis and decomposition, so as to enhance liver detoxification function. Furthermore, schizandrol extract can decrease the hepatitis patient transaminase levels, but also reduce the substance metabolic disturbance caused by injury to the liver poisoning and promote synthesis of liver intracellular protein to achieve therapeutic hepatitis effect[3]. The current study identified that schizandrol extract, schisandrin and schisandrin B can effectively reduce the damage of D- galactosamine on liver cells, inhibit the alanine aminotransferase (ALT), aspartate aminotransferase (AST) and monoamine oxidase (MAO) activity, and reduce malondialdehyde (MDA) content, to repair liver cells damage. Schisandrin and schisandrin B is the main active constituents of Schisandra block of liver cells injury. Schisandra chinensis(Turcz.) Baill can markedly resist liver injury of mice caused by $\mathrm{CCl} 4$. There are obvious protective and repairing effects of intraperitoneal injection of carbon tetrachloride acute liver injury in mice. Extraction of Schisandra chinensis(Turcz.) Baill on therapeutic effect of acute liver injury induced by CCl4 in mice, the results discovered that it can significantly reduce the content of ALT in serum, AST, alleviate the pathological damage, and repair effect of microstructure [4].

Hypoglycemic effect. Schisandra chinensis oil can make proliferation of four alloxan model mice islet B cells, increase the serum insulin levels, and lower glucagon levels. Schisandra oil can decrease MDA content, increase the activities of superoxide dismutase (SOD), improve the ability of antioxidant and scavenging oxygen free radical, reduce the level of lipid peroxidation; and also the 
expression of GLUT4 mRNA is enhanced in muscle tissue, so it improve the glucose transport capacity, reduce blood glucose, and ultimately achieve the effect of the treatment of diabetes [5].

Schisandra chinensis(Turcz.) Baill and other medicine composition mixture played a protective role in early kidney injury of diabetic rats. It could reduce the discharge of serum biochemical indexes of rats with diabetic nephropathy, decrease the urine protein content, and reduce the renal tissue pathology damage, furthermore, it made that fasting blood glucose is slightly decreased, so this confirmed that it has a good curative effect in the clinical treatment of diabetes.

Schisandra chinensis(Turcz.) Baill from molecular level to improve insulin sensitivity, the sensitivity of cells to insulin is improved via the activation of PPAR- signal pathway of gamma, this effect has been verified in the experiment.

For the high blood glucose four alloxan induced diabetic mice and adrenaline caused by isolated from Schisandra chinensis, alpha glucosidase inhibitors have obvious hypoglycemic effect, and significantly enhanced the amount of glucose tolerance in mice[6].

Anti-oxidant and anti-aging effect. The effective components of the medicine has anti-aging, antioxidant effect, such as schisandra fruit extract, Schisandra chinensis Turcz and crude polysaccharide could increase the activity of antioxidant enzymes in brain, liver and other tissues, such as superoxide dismutase (SOD), remove peroxidation by-products of malondialdehyde (MDA), lipid peroxide formation with homogenates significantly inhibited animal major organ. Schisanhenol could directly capture oxygen free radical damage of myocardial mitochondria, oxygen free radical and adriamycin against rats caused by free radical, protect and $\mathrm{Fe} 2+-\mathrm{Cys}$ induced rat brain synaptosomes [7]. In addition, Schisandra chinensis crude polysaccharide can significantly improve the ability of anti fatigue and hypoxia tolerance in mice, enhance the phagocytic function of reticuloendothelial system in mice, and increase the normal mouse immune organ weight; furthermore, its can decrease the serum of aged rat lipid peroxide (LPO) content [8].

Another study showed that Anti oxidative therapy through the large dose of hydrocortisone acetate induced oxidative balance disorder mice, discovered that Schisandra can significantly increase the SOD, GSH PX in serum (GSH-Px) activity, reduce the MDA, alleviate the oxidant antioxidant balance disorders[9].

The effects of immune regulation. Schisandra chinensis polysaccharide has immuno exciting function better. Schisandra chinensis polysaccharide regulate the body's immune function of lymphocytes, macrophages by reticuloendothelial system function. Effect of Polysaccharide from Fructus Schisandrae on normal mouse peritoneal macrophage, the research discovered that the high dose group of Schisandra chinensis polysaccharide can significantly improve the phagocytic index and phagocytic percentage of macrophage, and the hemolysin and hemolytic plaque forming a clear role in promoting. Schisandra as well as promoting lymphocyte transformation function can promote lymphocyte DNA synthesis, increase the lymphoblastoid cell generation and enhance the immune function of spleen. And Schisandra chinensis against allogeneic tissue transplantation rejection, immune enhancement of adrenal cortical hormone inhibition[10].

Ginseng Schisandrae decoction can effectively prevent asthma mice, its mechanism may be improving asthmatic reaction in mice through the influence of hematopoietic cells in CD34+ to the way of eosinophils and airway inflammation to the process of transformation. Extraction of crude polysaccharide from Schisandra chinensis in the residue with enhanced immune effect is very good. Compared with Schisandra chinensis and Schisandra, removal of protein polysaccharide and without removal of protein crude polysaccharide enhanced the phagocytic activity of peritoneal macrophages and the effects are very significant, while the South polysaccharide only in large doses can enhance the phagocytic function of celiac macrophage, and there is a dose-response relationship. Nonetheless, these two kinds of Polysaccharide from Schisandra can improve serum hemolysin level in mice[11].

Anti-tumor effect. Schisandra chinensis polysaccharide has a promoting role in thymus, spleen hyperplasia, tumor bearing S-180 mice solid tumor growth inhibition, and had a synergistic effect on tumor chemotherapy. Inhibition of Fructus Schisandrae polysaccharide anti-tumor effect may be through the promotion of tumor cell apoptosis, activation of immune cells to promote the realization of 
intratumoral and peritumoral inflammatory reaction[12]. Schisandra chinensis polysaccharide can reduce the rate of chromosome aberration, micronucleus rate of inhibition, with anti mutagenic activity, and has inhibitory effect on transplanted H-22 tumor and S-180 solid tumor. Expression of the intervention effect of Schisandra chinensis polysaccharide on $\mathrm{H}-22$ tumor bearing mice serum immune related cytokines significantly, the level of IL-2 expression is up-regulated in serum, and no obvious regulation effect on IL-10 and VEGF. Description of Fructus Schisandrae polysaccharide could inhibit transplanted H22 hepatoma in mice by up regulating the expression of IL-2 on the growth of. 4 different chemotherapy drugs anticancer mechanism applied alone or schisandrin B and every kinds of chemotherapeutic drugs combined application, compared their effects on tumor multidrug resistance cell line MCF27 /MDR1, results showed that schisandrin B sensitization to the 4 drugs have proved schisandrin B could be a tumor multidrug resistance reversal agents. Schisandra chinensis on aflatoxin Bl induced rat hepatic precancerous lesion, gamma glutamyl transpeptidase positive hepatocyte growth range has obvious inhibitory effect[13].

The role of the central nervous system. Effect of Schisandra chinensis on central nervous system clear, has obvious sedative and hypnotic effects, the dose showed a certain correlation, and does not produce drug dependence. The ethanol extract of Schisandra, Itsumi Kokosu, B, vitamin C and schisandrin B can obviously prolong the sleeping time of pentobarbital sodium, can enhance mice Nembutal subthreshold dose induced sleep sleep effect on mice sleep, suprathreshold dose of sleeping time prolonged. The crude and processed products of Fructus schisandrae chinensis has sedative and hypnotic effects, and the processed products effect is stronger than the crude, which wine is better than vinegar products. The ethanol extract of fruit kernel had the anticonvulsant action, and schisandrin had the similar effect.

Schisandra chinensis polysaccharide in reaches a certain concentration, has inhibitory effect on potassium antimonyl tartrate writhing reaction in mice, it could alleviate the pain behavior in rats induced by formaldehyde, showing a dose-response relationship, that of Fructus Schisandrae polysaccharide has significant inhibition effect on the two kind of pain, nevertheless, the analgesic mechanism of action is unclear[14].

Bactericidal effect. Schisandra can inhibit the intestinal tract of harmful bacteria, including Pseudomonas aeruginosa, Staphylococcus aureus, Salmonella typhi, Bacillus dysenteriae, Candida albicans and other intestinal conditional pathogenic bacteria. Furthermore, pathogens have a good inhibiting growth effect, but its mechanism is not clear. At the same time, Schisandra can promote the growth of beneficial bacteria to balance intestinal micro ecological balance. Thus, the antibacterial effect of Schisandra chinensis can be applied not only to the drug, can also play an important role in the aspects of food antiseptic[15].

\section{Prospect}

Schisandra Chinensis(Turcz.) Baill. has many pharmacological effects that relates to multiple system of the body. I believe that the scope of application of its medicinal will be broader with the in-depth study on the chemical constituents and pharmacological effects of Fructus schisandrae chinensis.

\section{Acknowledgements}

This work was funded by project "2013-199" supported by the Education Department of Jilin Province, project "201262503" supported by Sci-tech Department of Jilin City and project "20122082" the Healthe Department of Jilin Province.

Corresponding authors:

1. Liping An Email: alp960@ 126.com

2. Peige Du Email: dupeige2001@126.com 


\section{References}

[1] Fan Meihua. New progress in the study of Schisandra chinensis. Northwest Pharmaceutical Journal [J], 22(5):281-282, 2007.

[2] Yuan Haibo, Shen Zhongming, Yin Jianwei. Hypoglycemic effect of Schisandra chinensis qglucosidase inhibitors in mice [J], China Pharmaceutical Journal, 23(3):112-125, 2002.

[3] Xu Huiqin, ZhuQuan LiXiang. Inhibitory effect of 7 kinds of Chinese herbal medicine on the non enzymatic glycosylation in vitro generation of final product $[\mathrm{J}]$, Chinese herbal medicine, 33(2):145-147, 2002.

[4] LiLi, LiuGengtao. The protective effect of schisanhenol induced injury of rat cerebral mitochondria and synaptosomes on oxygen free radicals [J], Pharmaceutical Journal, 33(2): 81, 1998.

[5] Sun Wenjuan, LvWenwei,YuXiaofeng. Experimental study on the anti aging effect of Schisandra chinensis polysaccharide.[J], China Journal of Gerontology, 21(11):454, 2001.

[6] LiuJianhong, YaoNing WangXin. Experimental study of Schisandra chinensis induced oxidative balance disorder effects on hydrocortisone acetate.[J], Journal of clinical and experimental pathology Chinese, 15(5):69-70, 2009.

[7] Miao Mingsan, FangXiaoyan WangXin. Effects of Schisandra chinensis polysaccharide on immune function in normal mice [J], Chinese Chinese medicine science and technology, 10(2):100, 2003.

[8] SuMingwei, WangNaiping, XinHuawen. Research progress of pharmacological action of Fructus schisandrae chinensis [J], Chinese pharmacist, 12(7): 960-962, 2009.

[9] Qu Zheng Hai, Liu Xiao mei, Xie Ning. Study on the mechanism of ginseng Schisandrae Decoction in prevention of asthma in mice [J], Journal of integrated traditional Chinese and Western medicine China emergency, 15(4): 201-204, 2008.

[10]Qu Zheng Hai, Liu Xiao mei, Xie Ning. Study on the mechanism of ginseng Schisandrae Decoction in prevention of asthma in mice [J], Journal of integrated traditional Chinese and Western medicine China emergency, 15(4): 201-204, 2008.

[11] Lin RD, Mao YW, Leu SJ. The immuno-regulatory effects of Schisandra chinensis and its constituents on human monocytic leukemia cells [ J ], Molecules, 16(6): 4836-49, 2011.

[12] HuangLing, Chen Ling, Zhang Zhenlin. The immuno-regulatory effects of Schisandra chinensis and its constituents on human monocytic leukemia cells [ J ], Traditional Chinese Medicine, (3): 40, 2007.

[13] LiMingzhu, Wang Yanjie, Xu Fang. Experimental study of Polysaccharide from Schisandra inhibit tumor and mutation effects [ J ], Heilongjiang Journal of traditional Chinese Medicine, (3): 40, 2007.

[14] Yu He, Li Ji, LiShulian WangYanjie, Xu Fang.. Study on intervention effect of Fructus Schisandrae polysaccharide on $\mathrm{H} 22$ tumor bearing mice changes of serum Zn, Se levels [ J ], Information of traditional Chinese Medicine, 27(3): 25-26, 2010.

[15] Li, Li Ji, LiShulian Wang Yanjie, Xu Fang.. Study on intervention effect of Fructus Schisandrae polysaccharide on $\mathrm{H} 22$ tumor bearing mice changes of serum $\mathrm{Zn}$, Se levels [ J ], Information of traditional Chinese Medicine, 27(3): 25-26, 2010. 